\title{
Y a-t-il des traces du sentiment de l'identité canadienne dans la BD québécoise?
}

\author{
Mira Falardeau \\ Chercheure indépendante
}

Parler de l'identité canadienne dans une forme d'expression québécoise revient à questionner les fondements de l'identité québécoise, avec toutes les oppositions, pour ne pas dire les contradictions inhérentes au propos. Notre postulat soutient que ces deux identités se recoupent, même si dans son discours, la culture québécoise se distingue habituellement par sa différence et sa fierté de l'être. Dans les faits, les deux cultures sont les plus souvent imbriquées, l'une faisant partie de l'autre, aussi bien du point de vue géo - politique que culturel. La BD, art par excellence du symbole et de la litote, a su synthétiser ces points de vue et à travers six œuvres sélectionnées chez six grands artistes de la BD québécoise, échelonnées de 1900 à nos jours, nous tentons ici d'éclairer ces tiraillements et de démontrer comment ces mouvements parfois antagonistes donnent lieu à une parole dynamique et représentative.

Baptiste, le cocasse diplomate international, Onésime et les ruraux de Chartier, le super antihéros loufoque Capitaine Kébec, le Carcajou fripon amérindien de Laniel, la citadine solitaire du métro de Doucet, enfin Paul face à la société québécoise, tous à leur façon sont les chantres d'une façon de voir qui nous réunit à travers nos divergences. De notre projecteur, nous éclairerons tout d'abord leur tonalité de base. Puis, dans le sillon ainsi creusé, nous soulignerons la fibre particulière que chaque œuvre vient flatter et éventuellement, les ambiguiités du discours de ces grandes figures de notre imaginaire collectif.

La première œuvre abordée est Les Aventures de Ladébauche autour du monde (1910) du premier grand bédéiste québécois, Albéric Bourgeois (1876-1962). Bourgeois publie Les Aventures de Ladébauche autour du monde (1910) d'abord dans La Presse, puis en fascicule qui aura un immense succès. Narration comique d'un "habitant", autrement dit un paysan, face aux grands de ce monde, Les Aventures se lisent et se regardent à la fois, puisqu'il s'agit d'histoires en images, soit une suite d'images accompagnées de textes, les ancêtres de la bande dessinée. L'humour débridé est ici la force de base de cette œuvre, qui nous présente son héros cocasse jouant aux grands diplomates. Le contraste entre les deux univers crée un effet comique assuré, alors que Bourgeois descend la diplomatie internationale de son piédestal en la mettant aux mains d'un homme du peuple dont le nom de famille est déjà tout un programme! Ainsi, Baptiste va rencontrer Nicolas II de Russie (ill. 1.), l'Empereur Guillaume II d'Allemagne dans «Hërr Von Ladébaucheoffën chez Dillaume », le roi d'Espagne Alphonse 
XIII dans «El caballero y Ladébaucherez chez Alphonzo », et ainsi de suite.

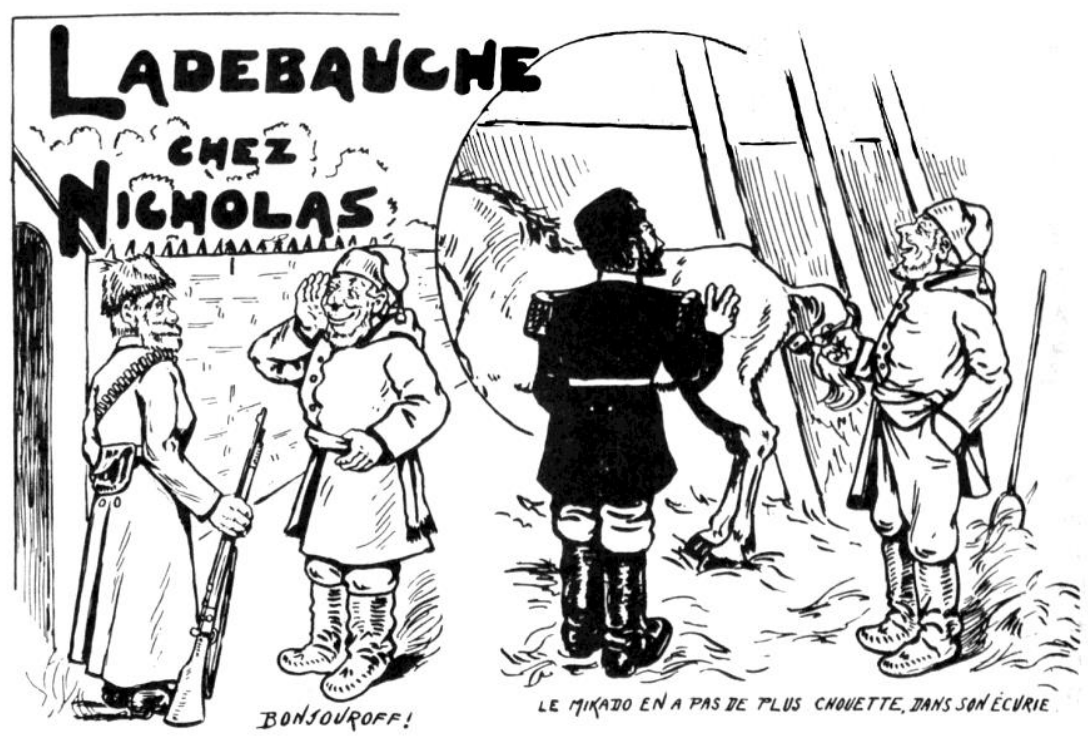

Figure 1 : Albéric Bourgeois (1910), « Ladébauche chez Nicholas » (25)

Cette façon de s'imaginer ainsi ambassadeur de sa nation auprès des grands lui confère une stature nationale. Il se donne lui-même la tâche de représenter son pays. Mais quel pays, au fait? Ladébauche est-il Canadien ou Canadien français? Rappelons que la dénomination de Québécois viendra beaucoup plus tard dans la terminologie, avec la montée du nationalisme québécois dans les années 1960. Il se dénomme luimême "canayen", ce qui n'est qu'une formule populaire, mais parfois « canayen-français ». Quand il va voir le roi du Royaume-Uni Édouard VII, celui-ci lui confie la tâche de réconcilier les Canadiens entre eux : «Quand tu seras rendu chez vous, dis à mes Canayens de cesser de se chicaner entre eux, ça paraît mal à l'étranger. » ("Ladébauche chez le roi », 19). Ainsi donc, ses interlocuteurs le voient comme un ambassadeur national et, par le fait même, lui en confèrent la responsabilité. Ladébauche, Canadien français ou Canadien?

"Le nationalisme canadien émerge du XIXe siècle au sein d'un peuple fortement majoritaire dans un territoire défini, le Bas-Canada (la province de Québec), mais politiquement et économiquement soumis à une minorité conquérante qui s'en distinguait à la fois par l'origine, la langue et la religion. » (Canet 138), Mais les Canadiens français eux-mêmes, peuple catholique, rural et parlant français qui habitait à l'origine sur les rives du fleuve Saint-Laurent, ont de la difficulté à se percevoir et à se 
définir durant tout le $20^{\mathrm{e}}$ siècle, coincés entre trois grandes cultures, les cultures française, américaine et anglaise. Ils répliquent avec un nationalisme qui a ses côtés positifs et négatifs.

«(Le nationalisme canadien-français)... emprisonne le passé canadien-français dans une interprétation à sens unique, dans un système de pensée qui accentue chez le Canadien français les ressentiments qu'a créé en lui son statut minoritaire ». (Falardeau, La dualité 32). C'est tout à fait ce "canayen» qui va aller faire la leçon tout autour du monde, en rabaissant les grandeurs des uns et des autres. Bourgeois réinterprète ici un héros existant, Baptiste Ladébauche, ébauché d'abord par la plume d'un grand journaliste du $19^{\mathrm{e}}$ siècle, Hector Berthelot, dans ses différents journaux satiriques dont le plus connu fut Le Canard (1877-1957). Puis, un dessinateur d'histoires en images, Charlebois, l'a transposé à La Presse. C'est donc un héros bien rodé, qui a déjà un caractère typique que reprend Bourgeois. Homme-orchestre, Bourgeois joue de toutes les cordes de l'humour: tour à tour bédéiste, narrateur d'histoires en images, caricaturiste, puis par la suite scénariste pour la radio et même auteur de comédies musicales, il va créer à travers son héros et sa femme, Baptiste et Catherine Ladébauche, deux personnages représentatifs de l'ensemble de la société québécoise : urbains, ils ont tout de même gardé des habitudes vestimentaires et les mœurs de la campagne, tels entre autres, leurs habillements caractéristiques des vêtements des colons. Baptiste Ladébauche porte sa tuque à longueur d'année de même que sa ceinture fléchée, faite de laine grossière tressée, typique des Canadiens français. Or, tout le $19^{\mathrm{e}}$ siècle a vu la montée d'une élite bourgeoise, urbaine et d'affaires canadienne-anglaise alors que la minorité canadienne-française restait fondamentalement rurale (Canet 162). Avec l'industrialisation massive du début du $20^{e}$ siècle, la dynamique évolue et nombre de villes de la Province de Québec sont prises d'assaut par les habitants de la campagne. Ladébauche synthétise le choc des deux styles de vie.

Rappelons pour mémoire que Bourgeois est le premier bédéiste francophone au monde (Falardeau, "La BD francaise") avec sa célèbre BD en couleurs Les Aventures de Timothée (La Patrie, 1904). Il passe à La Presse en 1905 et y inventera dans ses BD une foule de personnages, dont Toinon, Zidore, La famille Citrouillard, mais le plus célèbre est sans conteste Baptiste Ladébauche. Bourgeois restera à La Presse jusqu'en 1955 comme caricaturiste.

Il promène donc son héros Baptiste chez tous les grands de ce monde, et aussi de son pays, pour leur faire la leçon sur leur gouvernance, avec son franc-parler et sa gouaille traditionnelle. Les descriptions de ces rencontres sont complètement loufoques: il découvre le roi PierreEmmanuel en train de pêcher la barbotte et le roi Christian en train de recoudre ses boutons de pantalons! Ladébauche amène ses lecteurs en Chine, au Japon, en France, en Italie, en Espagne, en Allemagne, en Russie, 
au Danemark, en Turquie, puis les ramène en Amérique du Nord en passant par la Maison-Blanche. Que trouve-t-il lorsqu'il rend visite à Émile Loubet, président de la France? «Je file chez Émile que je trouve en train de se hacher une pipe de tabac sur le coin du piano pendant que sa femme reprisait ses chaussons et se berçait auprès du poêle en surveillant une crêpe au lard qui se laissait cuire sans dire un mot. » ("Ladébauche chez Loubet»18).

À la fois par bravade et par dérision, Ladébauche se dépasse luimême, se surpasse, devient plus grand que nature et l'illusion est si forte qu'il semble se prendre à son propre jeu. Mais en fait, il garde toujours les pieds sur terre et l'idéologie d'un socialisme naissant transparait dans ses commentaires. Quand on lui demande s'il veut être roi lui-même, il répond: «Ouais, je vous dirais ben franchement, ça m'a jamais tenté plus que rien, j'aime ben mieux être homme de chanquiers (chantiers); on sait ben, comme de raison, que c'est moins chouette, mais on est plus fier et plus indépendant, on gagne sa vie au lieu de sucer l'argent du pauvre peuple pour s'offrir des habits dorés et des beaux palais. » («Si j’étais roi » 138).

Laissons à Ladébauche la parole de la fin : Ladébauche, Canadien avant tout, comme il le raconte lui-même de sa langue verte, rapportant un échange avec le « Mikado, fils du Soleil à Pékin (sic)» :

Certainement, qu'y dit, mais d'éousque vous sortez?

De Trois-Rivières, bout de corde!

Yousque ça se trouve, ça?

Au Canada, batêche! » (Bourgeois 23)

L'ambiguïté entre les deux identités se poursuit chez les deux héros suivants, Onésime et Capitaine Kébec, emblématiques de la BDQ : à la fois Américains, Canadiens, Canadiens français et Québécois, ils condensent l'ensemble des points de vue. «Durant la période qui va de la Confédération à la Révolution tranquille, l'intention politique des Canadiens-Français du Québec, qui conditionne leur attitude au moment des élections, n'a surtout rien de simple ou d'univoque. Elle se maintient dans l'axe d'une ambivalence éprouvée que rien ne semble ébranler.» (Létourneau 65) En fait, Chartier représente un cas d'ambivalence assez particulier, reprenant au départ les thèmes des grands espaces des romans du terroir et de la colonisation, typiques d'une certaine vision romantique du Canada. C'est tout d'abord la fibre terrienne qu'il flatte dans l'ensemble de son œuvre, mais également le choc de la modernité. Même si le Canadien Onésime est essentiellement rural, à la façon des premiers découvreurs de ce pays, il ne peut s'empêcher, durant sa longue existence de héros de BD qui s'étend de 1943 à 2002, de rencontrer plusieurs révolutions dont l'urbanisation de masse et l'avènement des nouvelles technologies. De fait, «tous ces changements provoquent un questionnement sur l'identité collective et surtout sur l'identité nationale. » 
(Langlois 4) À travers les hauts et les bas de la vie sur une terre, Chartier nous raconte en concentré la vie des gens qui doivent s'adapter à la vie moderne tout en restant profondément ancrés dans leurs habitudes ancestrales et leur rapport symbiotique avec la nature.

Albert Chartier (1912-2004) est sans conteste l'auteur de BD qui a publié le plus longtemps au Québec. Les déboires d'Onésime et de sa femme Zénoïde ont paru pendant 59 ans dans Le Bulletin des agriculteurs, de 1943 à 2002. Après ses études à l'École des Beaux-Arts de Montréal, Chartier se lance dans la BD avec Bouboule sur un scénario de René O. Boivin dans La Patrie de 1935 à 1937. Les maladresses du gaffeur rondouillard annoncent Onésime. Après un séjour à New York, Chartier crée le célèbre Onésime, bègue et maladroit. À partir de 1943, celui-ci parait régulièrement chaque semaine sans interruption jusqu'en 2002 dans Le Bulletin des agriculteurs. Deux recueils d'Onésime sont édités en 1974 et 1975 par les Éditions l'Aurore tandis qu'un troisième sort en 1983 (Bulletin des agriculteurs). Puis, inspiré à nouveau par le thème de la colonisation des grands espaces, Chartier publie Séraphin, dans Le Bulletin des agriculteurs de 1951 à 1970, d'après un scénario de Claude-Henri Grignon.

L'histoire d'Onésime, cet habitant de la région rurale de Lanaudière, à l'est de Montréal et de sa femme Zénoïde est représentative de l'ensemble des ruraux et c'est une des raisons de son succès. Tous les gags d'Onésime ont la même structure : ils se déroulent sur une page de 6 à 10 cases et la dernière case est celle du gag final. S'il a débuté durant ce qu'on a dénommé « la grande noirceur», une époque où le Québec était encore régi par une mentalité ultra religieuse et des politiques conservatrices, on peut voir qu'Onésime a traversé la Révolution tranquille des années 60-70 et tous ses bouleversements sociaux et culturels à sa façon. En fait, Onésime résiste à la modernité, mais sans en avoir l'air.

«(Durant la période de l'après-guerre), le hiatus entre l'idéologie prônant un Québec traditionnel et rural reposant sur le triptyque languereligion-tradition et la réalité socio-économique d'un Québec fortement industrialisé, urbanisé et informé était devenue insoutenable ». (Létourneau 75) C'est pourtant l'une des caractéristiques de ce héros que son immobilisme. "Onésime, Zénoïde et les autres n'évoluent pour ainsi dire pas au fil de la série et des années. » (Carpentier 76) Durant ses 60 années de publication, il ne changera pour ainsi dire presque pas de mentalité, ce qui est en soit un exploit. S'il va à Expo 67, sommet des avancées technologiques et de la modernité, Onésime en revient fatigué («Vite sur les patins », juin 1967)! S'il danse, ce sont des danses carrées, typiques des campagnes («Un pied de nez aux U.S.A.», janvier 1963). Bref, tout changement à sa routine de paysan se traduit par une blague qui finalement insiste sur les valeurs de la tradition ou de la bonne vie à la campagne. 
Onésime est un personnage essentiellement comique. Le plus souvent victime des évènements, il termine généralement la page dans une situation avilissante ou par une déconvenue. "Ces cas de comique par détérioration dont Onésime est la victime couvrent une grande partie des scénarios des aventures d'Onésime. » (Carpentier 81) Tandis que sa femme Zénoïde s'en tire plutôt bien, quand ce n'est pas elle qui triomphe de son mari! On pourrait la considérer comme plus à l'avant-garde que son mari, entraînée par l'avancée du féminisme qui se développe durant tout le $20^{\mathrm{e}}$ siècle, mais plus particulièrement après la Révolution tranquille. Elle représente dans son essence ce personnage de la mère omnipuissante, typique du roman canadien-français, dont le schéma général est souvent celui de «l'aliénation du père et l'omnipotence de la mère ». (Falardeau "La dualité" 87)

La position d'Onésime par rapport à ses voisins anglophones est aussi totalement ambiguë : souvent ouvertement fâché contre le Canadien anglais ("Pas chanceux », septembre 1963), il est par contre en admiration béate face à la culture américaine, dont sa nièce est le plus pur reflet. Sa nièce américaine symbolise la modernité, la nouveauté face à laquelle Onésime perd ses moyens: il est bouche bée! Onésime semble donc représenter cet être déchiré entre deux idéologies, deux mondes, deux cultures, et c'est sans doute l'une des raisons de son succès et de sa longévité.

Hormis l'œuvre unique d'Onésime, la BD québécoise fait une «pause » - pause due en grande partie à l'invasion des comics américains qui vont s'approprier l'espace des journaux - entre les années des débuts du $20^{e}$ siècle avec Bourgeois et la naissance des fanzines des années 1970 durant le mouvement de la contre-culture. À cause de sa force symbolique, Le Capitaine Kébec de Pierre Fournier va devenir l'icône de toute la BDK, c'est-à-dire la bande dessinée kébécoise alternative de cette époque. 


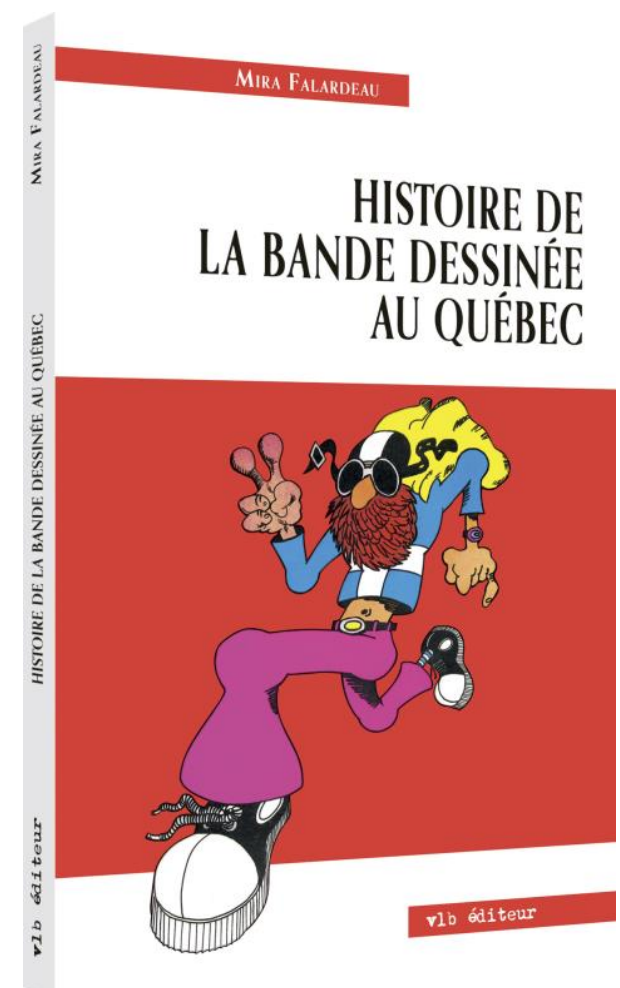

Figure 2: Pierre Fournier (1973), Capitaine Kébec, page de couverture Mira Falardeau, (2008) C) Capitaine Kébec MC et Pierre Fournier 2013.

Avec le Capitaine Kébec (1973), Pierre Fournier entre de plein pied dans le monde des comics et des superhéros, un monde que les bédéistes canadiens connaissent depuis plus longtemps. Provoqué par la Loi sur la conservation des changes en temps de guerre, l'âge d'or de la BD canadienne au moment de la guerre 1939-1945 a suscité la naissance des premiers super héros canadiens tels Nelvana of the Northern Lights (Adrian Dingle, 1945) et Johnny Canuck (Léo Bachle, 1942) qui auront droit eux aussi à la moquerie des antihéros canadiens. Mais cet univers n'est pas tant familier aux lecteurs de $\mathrm{BD}$ québécois, plus attirés par la BD franco-belge, aussi bien à cause de la langue que du fait que la dite $\mathrm{BD}$ est en pleine expansion au début des années 1970 avec l'explosion des titres de revues pour adultes, dont par exemple les délirants L'Écho des Savanes, Fluide Glacial, Métal Hurlant. Mais ce que Capitaine Kébec partage avec ses contemporains antihéros canadiens tels Captain Canada (Bernèche et Évans, 1972) (figure 3) et Captain Canuck (Comely, 1975-1981) tient au fait qu'il est comme eux l'illustration condensée de tout le sentiment de moquerie que les Canadiens éprouvent envers les vrais super héros américains qui symbolisent à leurs yeux le pouvoir excessif des Américains! Cet antihéros entreprend ici un dialogue tacite avec la BD de super héros loufoques dont il épouse les codes et les errances. 


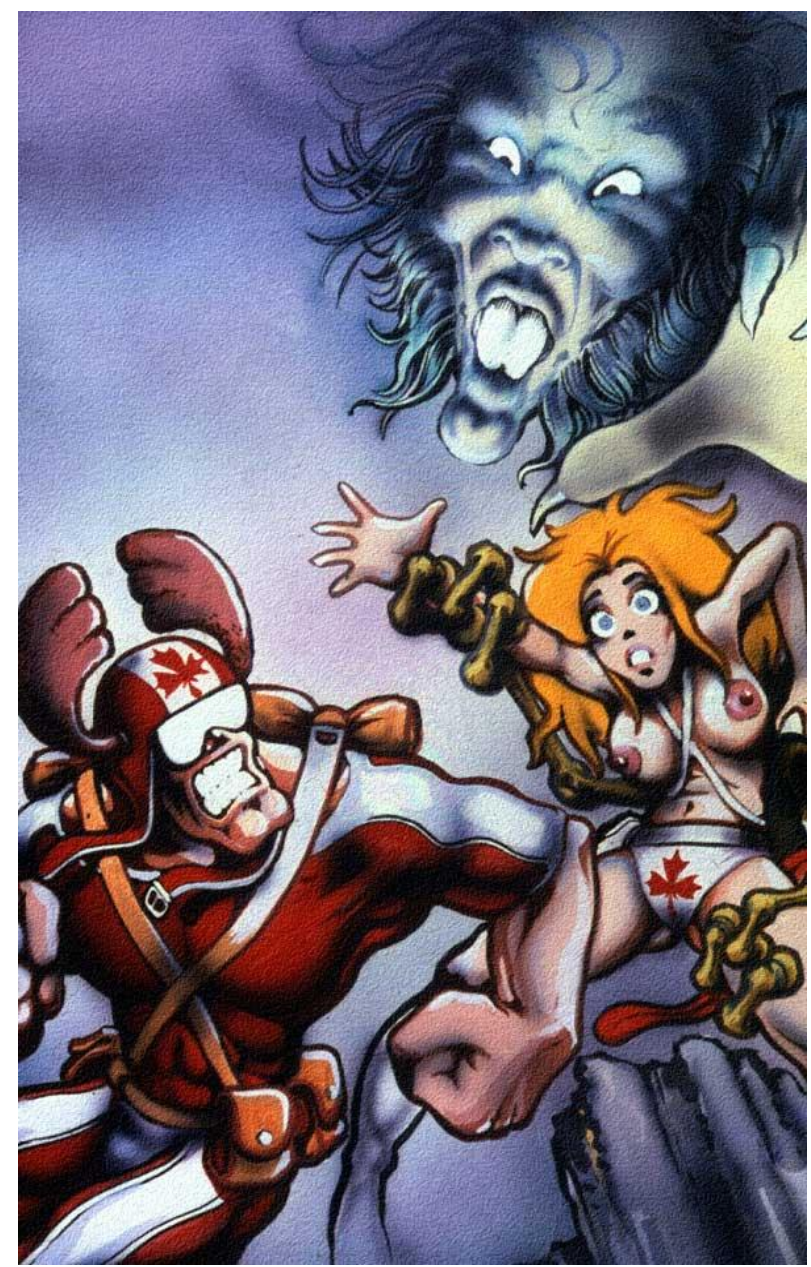

Figure 3 : Stanley Bernèche, Captain Canada, 1972. (C) Stanley Bernèche 1972.

Comment est né le Capitaine Kébec? En 1971, un projet de Perspectives-Jeunesse, programme fédéral de subventions, permet l'éclosion d'un fanzine particulier à Montréal, L'Hydrocéphale (2 numéros). Les Éditions de l'Hydrocéphale Entêté produisent des revues de petits formats à la façon des comic books américains, sur papier journal avec couverture couleur. Présentée de cette façon, la BDK a moins de succès que prévu, car les comic books ne font pas partie de la culture québécoise, tel que le soulignera d'ailleurs Pierre Fournier par allusion dans sa BD. Par la suite, la carrière de Pierre Fournier sera associée à celle de Réal Godbout dont il deviendra le fidèle collaborateur aux scénarios des héros Michel Risque (trois albums, 1981-1984) et Red Ketchup (trois albums, 1988-1994). Les deux séries sont reprises par les éditions La Pastèque depuis 2005.

Dans le seul comic book jamais publié avec ce héros mythique, il se décrit lui-même (Fournier 5) comme arborant un casque d'aviateur datant «du temps où il faisait de la moto », une vieille montre sans aiguille « juste pour la forme » et sa cape, formée d'une « serviette gratis sortie d'une boite 
de détergent mini-mousse", des lunettes de soudeur, et surtout son chandail de la Saint-Jean Baptiste, la fête nationale du Québec, où le drapeau fleurdelisé du Québec s'étale sur son torse. Bref, d'une allure totalement ridicule, le superhéros de pacotille est tout de même doué de super pouvoirs dont celui de voler. Suivant la logique des épopées des super héros, il lutte contre ses ennemis. Frogueman l'attaque de front. Cette espèce de créature bosselée mi-grenouille, mi-homme projette un liquide visqueux, de la soupe aux pois, allusions ouvertes aux qualificatifs moqueurs usuels que l'on donne aux Québécois : «French pea soup » et «French Frogs». La bataille classique se déroule dans un décor de toits urbains, où le capitaine reçoit l'aide de l'un de ses admirateurs, un jeune anglophone fou de comic books. Bel exemple d'intertextualité que nous sert ici Fournier: est-ce un message sous-entendant que les lecteurs francophones ont besoin des lecteurs anglophones de comic books vu qu'ils ne sont pas familiers avec cet univers de super héros? La cause de Capitaine Kébec reste nébuleuse jusqu'au moment où il fait face à son ennemi qui se présente comme un ancien policier en lui sortant une tirade sur les valeurs du passé, sapées d'après lui par des gens comme le capitaine, qui écoutent de la musique de sauvage, prennent de la drogue et ne respectent plus la loil Sauvé par le jeune anglophone, il tombe tout de même à la toute fin entre les griffes de son second ennemi que l'on voit tramer un enlèvement en parallèle depuis le début de l'histoire. Celui-ci a tout du fou classique : il est âgé, très riche, en chaise roulante, et il vit dans un vieux couvent désaffecté où il a aménagé un laboratoire qui évoque une salle d'opération avec, à sa solde, un serviteur gigantesque! La dernière case nous annonce la suite à Rivière des Prairies, quartier de Montréal au bord de la rivière du même nom qui sépare Montréal de la Ville de Laval, et la rencontre avec une «légende vivante» — sans la nommer — venant du Grand Nord, mais ce comic book ne sera suivi que de trois petits épisodes. Le premier est une planche hommage sous la plume de Fern (Fernand Choquette) dans L'Illustré no 8 (1974) qui raconte la rencontre dans un bar entre le Capitaine Kébec et le capitaine le plus populaire de la BD francophone, le capitaine Haddock, célèbre compagnon de Tintin de la série d'Hergé. Mais la succession du célèbre auteur interdisant toute reproduction des personnages d'Hergé, la planche n'aura aucune survie possible. Enfin, le Capitaine Kébec refait deux apparitions dans la revue de BD Titanic en 1984 (nos 5 et 6), mais sans suite : sa vie idéale aurait dû se dérouler dans une revue du type des comic books. Mais ceux-ci n'ont pas de pendant francophone, et ce sont les revues de bandes dessinées classiques, à la suite de l'école franco-belge, qui vont essaimer en français au Québec, telles Croc (1979-1995), Safarir (1987-) et Délire (1996-). Obéissant dans sa narration à la structure des comic books de supers héros canadiens et américains, Capitaine Kébec leur répond en quelque sorte comme par écho et c'est dommage que l'incursion ait été si brève! 
Le cliché de l'antihéros n'est pas spécifique au Canada, peu s'en faut, mais il prend ici une tonalité toute particulière et son succès dépasse de loin toutes les espérances de son auteur, qui par la suite, n'a pu retrouver l'état de grâce des débuts et s'est tourné vers d'autres œuvres. Pourquoi une telle notoriété pour un antihéros somme toute assez dérisoire : ses aventures ne sont qu'une répétition de clichés éculés, et sa vie est l'une des plus courtes de la BD? Tout tient en un mot : il parlait à l'inconscient collectif des Québécois. Enfin, une icône de l'autodérision, typique d'un peuple minoritaire!

Si Les Aventures de Ladébauche est le premier recueil d'histoires en images publié au Québec, en 1910, reprises peu de temps après leur parution dans le journal, un peu à la façon des comic books, Carcajou, le glouton fripon (1982), de Grozoeil (pseudonyme de Christine Laniel) est le premier album de BD cartonné en couleur signé par une femme au Québec, et à ce titre, il mérite notre attention. Avec un style graphique s'inspirant de l'art amérindien, Carcajou raconte une légende animalière issue de la culture autochtone, culture qui imprègne l'ensemble du pays à travers ses dizaines de peuples et de tribus différentes.

La bande dessinée et l'art autochtone ont rarement été réunis, et nous avons droit ici à des légendes attikamèques, relatives aux Amérindiens Abénakis, adaptées par l'anthropologue Claude Lachapelle. Laniel les a illustrées de sa ligne graphique marquée par des traits appuyés et une ornementation débordante. Christine Laniel a une formation en Arts plastiques et en audiovisuel. Elle débute la BD dans les fanzines des années 1970 à Montréal. Son style âpre évoquant la rugosité des gravures sur bois se rapproche déjà des arts primitifs lorsqu'elle signe ses premières BD dans la revue Baloune en 1977. Elle publie dans les journaux les plus engagés tels Le Temps Fou, Mainmise et La petite Gazette Hebdo. Dès 1980, elle quitte les collaborations régulières pour se consacrer à la réalisation de l'album Carcajon, ce qui deviendra l'œuvre de sa vie, puisqu'elle a par la suite abandonné la production de BD.

Carcajon va puiser au cœur même de la spiritualité amérindienne, commune à l'ensemble des communautés autochtones du Canada. Animistes, les auteurs de ces légendes établissent un lien direct entre les diverses formes de vie terrestre et les forces de la nature. "Ils croyaient qu'avant le déluge existait une communication directe entre notre monde et le monde supérieur que nous appelons ciel, que les astres, les forces occultes et les animaux prenaient forme humaine à volonté et que d'autre part, des hommes privilégiés pouvaient obtenir de puissances surnaturelles des pouvoirs exceptionnels. » (Mélançon 2)

L'album est constitué de quatre légendes. Un texte de présentation les précède où l'on apprend que le carcajou est un animal réputé rusé et 
même coquin, d'où l'appellation de "fripon", car il déjoue les pièges et arrose ses adversaires d'un liquide malodorant. Le carcajou, aussi appelé glouton, est un mammifère qui ressemble à un petit ours ou à un gros chien, et à cause de son appétit vorace et de sa vivacité, il est appelé «l'animal le plus féroce du Grand Nord». Ses habitudes défensives ont incité les autochtones à le qualifier de petit coquin, comme s'il était comique de façon délibérée! Il y a ici un mélange des genres qui peut étonner le lecteur occidental, peu habitué à marier humour et spiritualité! Par cet anthropomorphisme qui est une des caractéristiques principales de la spiritualité amérindienne, les narrateurs ont donc projeté sur cet animal des qualités intrinsèquement humaines!

Dans la première légende illustrée, "Carcajou et la moufette », Carcajou, après avoir tué la mouffette, va se laver dans l'eau de la mer et ainsi la rend volontairement salée à cause du liquide malodorant projeté par la mouffette! On y avance que l'origine du sel de la mer provient de cette blague de Carcajou! Le ton et les allusions aux parties intimes de l'animal sont étonnants pour le lecteur occidental, mais renforcent la sensation d'une tonalité différente, réservée aux seuls initiés, où les codes sont particuliers à un certain type de culture et étrangers aux lecteurs non avertis. Dans la seconde légende, "Carcajou et l'apprentissage du feu », il va à la chasse avec le renard et la chouette, dans un environnement de Grand Nord, et Carcajou, qui a de plus en plus les attitudes de l'humain, ayant quitté sa femme et ses enfants sous leur tipi pour aller chasser, se voit offrir par le renard le cadeau du feu. Ce renard a une connaissance magique des éléments naturels, il incarne en fait le pont entre les forces surnaturelles et les êtres vivants sur la terre. Enfin, dans les troisième et quatrième légendes, Carcajou chasse et fait cuire des outardes puis des perdrix. Ses méthodes de cuisson et de protection de son gibier sont pour le moins étonnantes, les déjections jouant un grand rôle dans ses méthodes de camouflage! Le ton comique des légendes indique que les autochtones s'amusent énormément en les racontant!

Dans ces légendes illustrées par Laniel en couleurs vives, Carcajou est présenté soit à quatre pattes soit debout comme s'il marchait sur ses pattes de derrière et cette démarche lui donne une attitude humaine, renforcie par le fait qu'il parle. Mi-homme, mi-bête, le carcajou de Laniel évolue au fil des histoires de la position quadrupède à la position humaine, ce que la figure 4. montre bien. L'animal, au rythme des diverses légendes, se rapproche insensiblement de l'humain qui le raconte. Selon les croyances autochtones, non seulement il parle, mais il parle à d'autres animaux, comme si tout le spectacle de la nature animale était à l'image humaine, un immense dialogue sans fin entre les êtres vivants. Ici, les pérégrinations de Carcajou l'amèneront à être à l'origine de divers fruits et plantes, dans une vaste genèse ou tentative d'explication du monde. La narration de Laniel est dynamique, elle accélère parfois le rythme en traçant deux ou trois fois 
le même Carcajou dans une seule case. Elle opère un montage chronologique, ne dérogeant pas à la logique intrinsèque des textes, accompagnés de commentaires narratifs de personnages assis en retrait, comme hors de l'histoire, qui nous expliquent les notions de base de la culture autochtone.

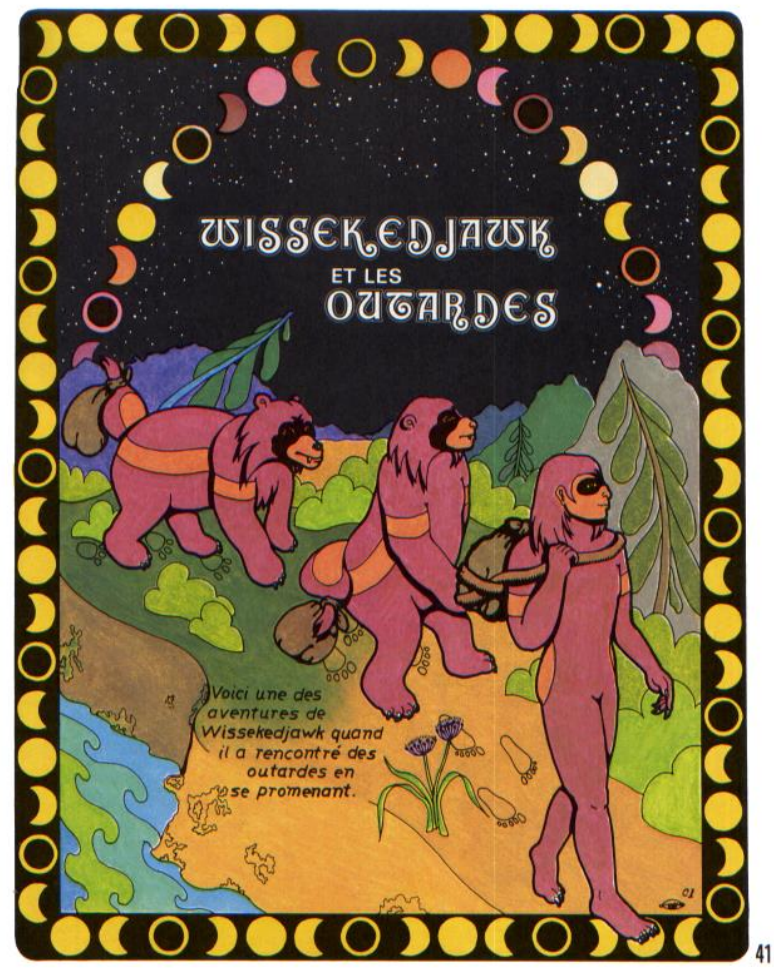

Figure 4 : (Laniel et Lachapelle 41). (C) Christine Laniel 1982

La nature est omniprésente, elle devient même un personnage à part entière dans la mesure où même les arbres parlent dans les légendes amérindiennes! À ce moment-là, Laniel leur ajoute tout simplement deux yeux, un nez et une bouche ainsi qu'une bulle pour leurs paroles! Curieusement, le petit rire intérieur que nous sentions en regardant Ladébauche auprès des grands de ce monde est le même que nous ressentons à la lecture du fripon Carcajou. Comme un sourire de complicité avec les auditeurs des légendes, qui semblent nous dire : "Vous voyez bien que nous sommes très forts, même si notre culture est méconnue? » En sous-entendu, l'on croit percevoir la fierté des peuples premiers face à la culture occidentale.

Dans un rappel des tonalités de base de l'art rituel autochtone, Laniel trace les contours des animaux d'un profond trait noir, et fait appel aux couleurs chaudes pour Carcajon et ses amis les animaux. Historiquement, l’iconographie amérindienne n'a occupé que des espaces 
personnels (les vêtements) tandis que l'art sacré nous a laissé très peu de traces, constitué essentiellement par les masques (Vastokas). Or les masques soulignent de façon appuyée les yeux et la bouche, de couleur souvent rouge et noire, à la façon des totems de l'art autochtone de la côte ouest (art Haida) du Pacifique. Le carcajou de Laniel évolue ainsi dans un décor quasi ritualisé où les couleurs primaires s'entre choquent, les chaudes teintes de rouge et de jaune des vivants se distinguant des couleurs froides des bleus et verts de la nature. Ce contraste visuel donne une perspective spirituelle aux formes des animaux. En mettant le langage de la BD au service d'une iconographie et d'une spiritualité autochtones, Laniel crée un pont bien nécessaire après des siècles de silence entre les cultures canadiennes officielles francophones et anglophones d'une part et les cultures des peuples premiers d'autre part.

Entre Laniel et Doucet, nous changeons subitement de registre, puisque, après la nature et les grands espaces, nous sommes ici en présence d'une mélopée urbaine étouffante, fable de l'anonymat et de la nonidentité. Missing est une nouvelle graphique de Julie Doucet (1965-) tirée du recueil de BD underground Dirty Plotte (1992).

D'emblée, on se sent dans une ville canadienne, ne serait-ce que par cette façon particulière dont Julie Doucet s'exprime, dans un anglais approximatif typique d'une francophone, son choix d'auteure. Comme elle le dit elle-même en entrevue (2010) : «Et les gens aimaient ma drôle de façon française d'écrire l'anglais ». Le titre très vulgaire de la série, "sale putain» en français, est d'ailleurs constitué des deux langues ("plotte» signifie putain en québécois), ce choix d'un bilinguisme assez particulier se superposant à un jeu de mots puisque que «plot» signifie «intrigue » en anglais, ce qui donnerait « sale intrigue » au sens littéral.

Cette narration underground urbaine est une œuvre qui frappe par sa polysémie et son discours dense. Un autre jeu de mots, avec «underground» cette fois, est cocasse puisqu'en fait, toute la scène se déroule dans le métro d'une métropole contemporaine. On se sent au cœur de la modernité, dans le ventre de la cité. Aucune inscription ne mentionne la localisation précise : sommes-nous à New York, comme dans la nouvelle précédente, à Montréal, la ville de l'auteure, à Toronto, l'autre métropole canadienne? Cette absence de point de repère reflète l'état d'âme de l'auteure qui se met elle-même en scène.

Dessinatrice alternative, Julie Doucet est la seule femme cartooniste québécoise qui ait acquis une réputation internationale. Sa carrière est multiforme et ses talents, polyvalents. Née à Saint-Lambert, dans la région de Montréal, elle étudie les Arts plastiques au Cégep du Vieux-Montréal et par la suite, à l'Université du Québec à Montréal où elle étudie les arts de l'impression. Elle s'adonne dès la vingtaine à la bande dessinée de fanzines. 
De 1988 à 1990, elle s'autopublie dans une série de fanzines au titre provocateur de Dirty Plotte (14 numéros), repris par l'éditeur anglophone d'avant-garde montréalais Chris Oliveros et sa maison Drawn \& Quarterly (1990 -), puis par Wimmen's Comix et dans la revue Weirdo de Robert Crumb (1943 -), père de l'underground américain. Il faut dire que son art parle ce langage "crumbien », à l'esthétisme à la fois intestinal et pornographique, dont le représentant montréalais le plus connu est Henriette Valium (pseud. de Patrick Henley, 1959-). Par son style, Doucet se situe dès ses débuts à la confluence des mouvements artistiques alternatifs américains et canadiens dont elle deviendra un peu malgré elle l'une des égéries majeures. Une quinzaine d'albums étonnants paraissent dans les deux langues jusqu'en 2000, dont My New York Diary (Drawn \& Quarterly, 1999, Chroniques de New York, Seuil, 2003) et Ciboire de criss (L'Association, 1996) et Changements d'adresse (1998). Puis, elle s'oriente vers les collages d'art et la lithographie. Elle est désignée en 2003 par le Beaux-Arts Magazine comme l'une des plus grandes artistes de la BD contemporaine. "J'étais comme une curiosité, une si jolie fille dessinant de si sales comics. » (2010)

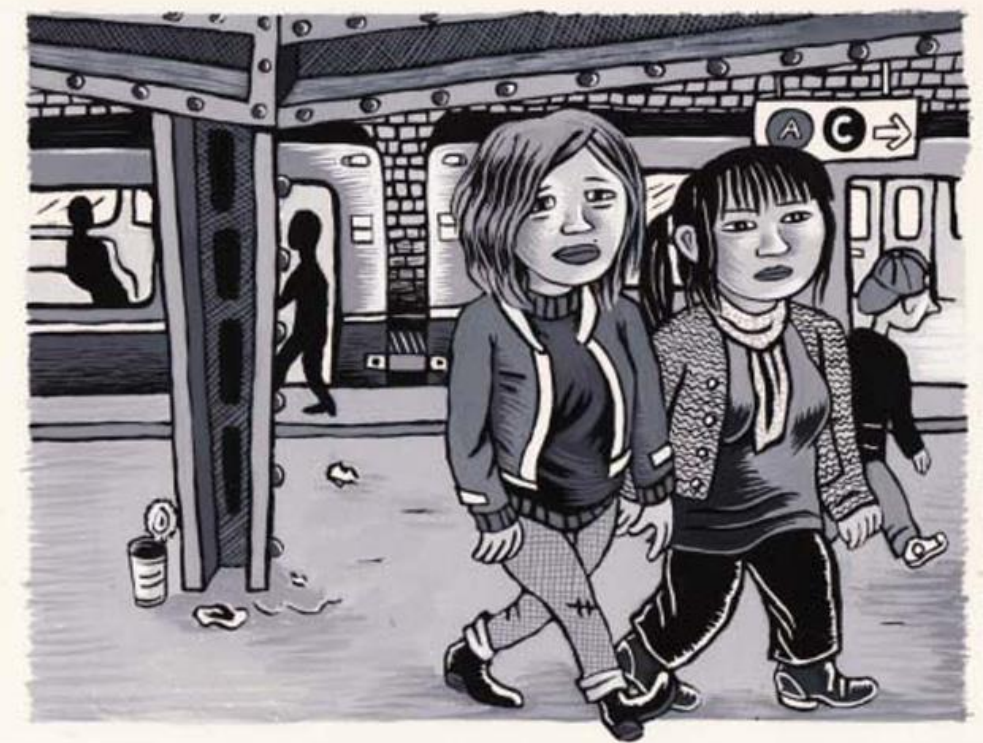

Figure 5 : Julie Doucet (1992), case 1. (C) Julie Doucet 1992.

Missing, an other bad dream s'ouvre sur cette image (Figure 5) de deux jeunes filles marchant dans le métro. La première est le « je » de l'auteure, la seconde est son amie Brigitte, d'origine asiatique, une allusion au caractère multiethnique des grandes villes canadiennes. Tout en déambulant dans cette atmosphère souterraine et enfermée, le personnage central perdra successivement trois personnes. La première est une perte de présence. Tout à coup, cette amie avec laquelle elle marche a disparu. Elle la cherche, revient sur ses pas, tourne une fois ou deux dans les tunnels pour réaliser qu'elle a bien disparu. Le surréalisme de Doucet, qui trouvera sa pleine mesure dans son art du collage où elle excelle depuis une dizaine d'années, s'exprime ici dans la symbolique des murs qui semblent avoir avalé l'amie 
disparue. À la façon des rêves où le décor semble parfois vivant. De même, les objets se répondent les uns aux autres par des liens oniriques, où les articulations sémantiques se font par automatisme. Ils parlent le langage de la destruction, un os, un éclat de vaisselle, une grille d'égout, une crevasse. Découragée, elle finit par se résoudre à abandonner la recherche alors qu'un second ami l'accroche soudain par l'épaule pour lui annoncer qu'un de leurs amis communs, Nicolas, est mort : celui-ci s'est sans doute suicidé en se lançant sous le métro, puisqu'on le voit passer sur une civière. Perte physique et émotive qui frappe le personnage principal de plein fouet. Mais le téléphone - un téléphone public, ce qui est assez surprenant et démontre que le personnage « habite » bien le métro — sonne et elle répond. C'est un troisième ami, John, qui voudrait la relancer pour un projet commun qu'ils avaient de se marier pour qu'elle acquière la nationalité américaine : trop tard, elle en rit maintenant, en lui demandant tout de même si c'est pour lui rendre service ou par amour qu'il lui redemande cette question. Puis subitement, le silence. L'appareil ne répond plus, elle est seule sur la ligne, à appeler et à appeler encore dans le vide : "Hello?, Hello?». C'est ici à la fois une privation physique et morale, car elle ne saura jamais si cet ami l'aimait. Fable minimaliste sur la présence et l'absence, Missing évoque le vertige face à l'anonymat de la grande ville, surtout dans ses lieux de transit comme le métro. Diverses cultures qui souvent se croisent sans vraiment se rencontrer évoluent dans ce rythme lent tel un chant triste en trois temps. Est-ce une allusion au multiculturalisme des métropoles? Le fait est que sur les trois absents, deux sont d'origine étrangère : la première, Brigitte est asiatique, le troisième, John, est Américain.

Contrairement à la ligne expressionniste et aux propos pornographiques des autres chapitres de Dirty Plotte, Doucet parle ici un discours épuré, à la ligne ample et presque poétique. La narration est classique, les unités de lieu, de temps et d'action sont respectées et même honorées dans ce tryptique où Doucet se surpasse. Les plans sont dynamiques, les champs/contrechamps appliqués en rythme, tout le vocabulaire de la $\mathrm{BD}$ est maitrisé avec une agilité qui accentue les effets dramatiques. Car c'est à un spectacle de véritable drame que ces noirs et gris foncés nous invitent, les textures fortement marquées sur les murs et les planchers, à la façon habituelle de l'artiste, renforçant encore l'effet suffocant de ces espaces clos. Étouffant dans la case, dans le métro et dans sa vie, le personnage central nous invite à partager sa solitude dans l’immensité de la grande ville. Missing, un condensé de la ville, côté négatif.

Missing, est une courte nouvelle graphique qui annonce déjà tous les éléments de la narrativité qui seront propres à cette grande artiste, à la confluence de trois cultures. Francophone qui écrit en anglais «avec l'accent», elle joue sur plusieurs tableaux et son propos fait la jonction entre l'underground américain et canadien et l'art de la métropole montréalaise. 
La vision également urbaine de Rabagliati (1961-) dans Paul à Québec (2009) est aux antipodes des sentiments de Doucet, puisque c'est la tendresse et la solidarité qui sont ici à l'honneur. Rabagliati accentue la position traditionnelle de l'artiste qui se situe toujours un peu en dehors de ce qu'il décrit. La distanciation prend ici une tonalité particulière, car il parle à la fois de sa propre société et de la société de l'Autre. L'Autre étant sa belle-famille, dont il se recule pour mieux l'observer.

Des sept albums publiés jusqu'à aujourd'hui, Paul à Québec est sans doute l'album québécois qui a fait le plus parler de lui. Récipiendaire du prix du public au Festival international de la bande dessinée d'Angoulême en 2010, il raconte avec beaucoup de sensibilité le décès du père de la conjointe de Paul, un triste évènement au sein de cette famille québécoise traditionnelle. Il ne suffit que de considérer cette vue à vol d'oiseau d'un quartier typique de la banlieue (ill. 6) où les piscines hors terre jouxtant les bungalows aux formes anonymes pourraient se retrouver sous n'importe quelle latitude nord-américaine.

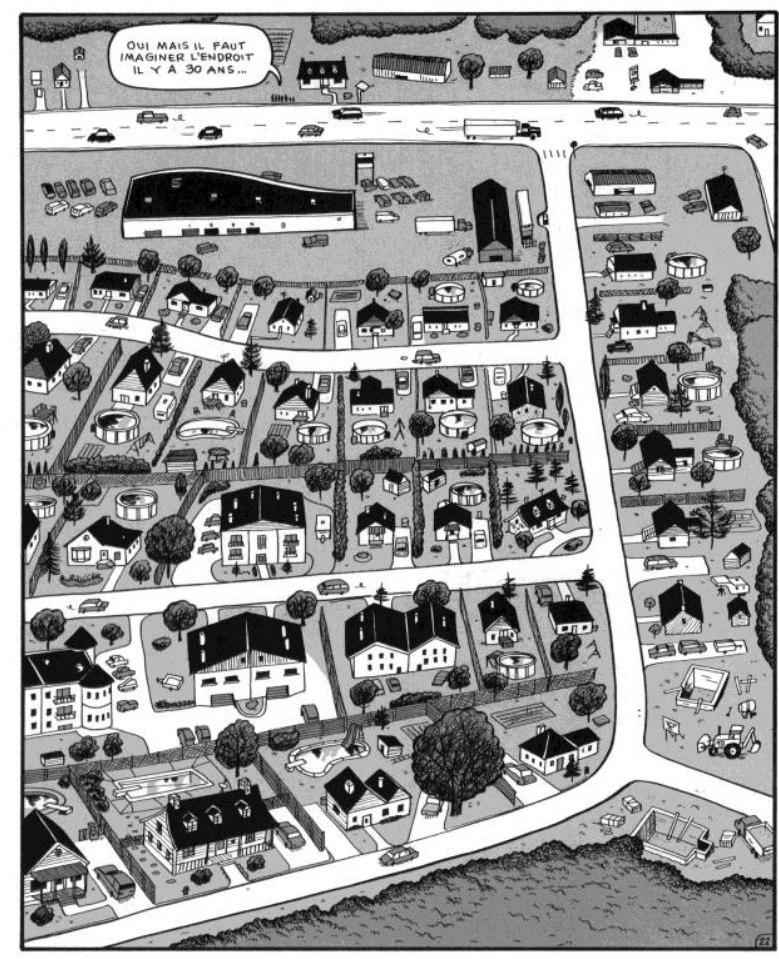

Figure 6 : (Rabagliati 28). @ Michel Rabagliati 2009 
Soulignons l'aspect hautement autobiographique de l'ensemble de l'œuvre de Rabagliati, qui a choisi délibérément sa propre vie et celle de ses proches comme matière première de ses récits, dans la lignée des romans graphiques des années 1980-1990. «Ce gars-là, c’est moi », dit-il dans une entrevue. "Les membres de sa famille, des amis et des connaissances habitent ses récits sous de faux noms. ", précise Louise Daveluy (32). Ici, c'est donc de sa belle-famille dont il traite dans l'intimité, et de leur traversée de l'épreuve ultime d'une famille face à l'agonie d'un proche.

La carrière de Rabagliati suit le parcours typique des auteurs de la nouvelle vague. Inspirées avant tout par sa propre vie, ses histoires de Paul sont dessinées d'un trait schématique. Paul à la campagne (La Pastèque, 1999) est traduit par Drawn \& Quarterly, Paul in the country, puis tous les autres albums à sa suite. Des traductions en plusieurs langues suivent, dont l'espagnol et l'allemand. Paul a un travail d'été (2002) gagne un Harvey Award. Les autres, Paul en appartement, Paul dans le métro, Paul à la pếche, Paul à Québec, Paul au parc (La Pastèque, 2004, 2005, 2006, 2009, 2011) poursuivent le récit de l'existence de ce jeune montréalais qui traverse sa vie en nous livrant ses pensées et en nous racontant ses hauts et ses bas.

Dans Paul à Québec, Rabagliati joue sur les deux "couches» identitaires superposées d'une manière astucieuse qui donne le ton de l'ensemble : au départ, on assiste au rassemblement de toute la belle-famille à l'occasion de la Fête nationale québécoise du 24 juin. Tandis que les jeunes proclament bien haut leur appartenance québécoise en trinquant à «l'indépendance du Québec», le beau-père de Paul s'interpose vigoureusement : «Not» pays, c'est le Canada! Pis not » province, c'est le Québec! C'est de même que c'est, pis c'est de même que ça doit rester » (14). Le clivage classique de nombreuses familles québécoises est ainsi présenté d'entrée de jeu: tous les jeunes adultes sont indépendantistes tandis que le «vieux » est fédéraliste. Puis, à la suite, dans cette première partie de l'histoire, nombre de traditions folkloriques québécoises sont évoquées : la cabane à sucre (36), les pères Noël déguisés (35), les desserts typiques (37), le fait de jouer aux «Indiens » (34). Dans la deuxième partie, de loin la plus longue, c'est la narration de la maladie du beau-père, de son agonie et de sa mort qui prend toute la place. À travers ses confidences à Paul, le beau-père se remémore sa vie au complet: le self-made-man typique parti d'une enfance dans l'extrême pauvreté pour monter à la force de son travail dans l'échelle sociale jusque dans les postes de direction d'une entreprise en alimentation. Paul-Michel y dessine la trajectoire d'un self-made-man à l'américaine, toutefois sans l'héroïsme souvent rattaché à ces parcours.

Il y a un regard particulier qui nous frappe dans le point de vue de Rabagliati. Cette perception nous est essentiellement personnelle, mais nous semble fondamentale pour bien comprendre son œuvre. Dans une 
entrevue (Deglise s.p.), Rabagliati parle de deux membres de sa famille (sa grand-mère et une grand-tante) qui sont nées en France. Même s'il est né au Québec, le fait qu'il provienne d'une famille issue de l'immigration fait qu'il a une vision à la fois de l'intérieur (québécoise) et de l'extérieur (européenne) de la société québécoise. C'est dans cet espace particulier qu'il se situe, analysant avec l'intérêt d'un entomologiste les structures familiales et sociales auxquelles il est confronté, qui ne sont pas celles de sa propre famille, mais bien celles de sa belle-famille qui joue le rôle de l'Autre. Il est à la fois proche et distant. On le sent se reculer hors du cercle tracé par cette famille dans ses rites, fêtes familiales, jeux, repas de groupe, comme s'il les observait par la fenêtre. L'artiste se fait ici sociologue par l'image.

Qu'y-a-t-il de voisin entre le personnage issu du peuple qui se moque des rois, le gaffeur malchanceux, le superhéros loufoque et l'animal mi-homme, mi-bête qui nous raconte ses sagas de la nuit des temps? Pas grand-chose de prime abord. Et pourtant, entre Baptiste, Onésime, Capitaine Kébec et Carcajon, il y a cette sorte d'humour qui est l'humour des petits face aux grands. Oserons-nous faire le saut pour comparer cette situation au sort du Canada, pays immense par sa superficie, mais non par sa population ou sa culture et qui se sent souvent bien écrasé par les grandes puissances qui sont aussi ses interlocuteurs privilégiés? La BD, par la condensation qu'elle opère, semble bien placée pour résumer en les caricaturant les complexes refoulés de générations de Canadiens et de Québécois face à leurs "mères patrie » l'Empire britannique, ou la vieille Europe, ou alors, leur voisin immédiat, les États-Unis, qui tous, dans des proportions variables semblent les écraser de leur outrecuidance, de leurs lois et de leurs cultures qui souvent nient ou étouffent leurs identités.

Baptiste et Carcajou. D'une part, l'humour populiste devant le snobisme des aristocrates et des héritiers du pouvoir de droit divin, puis le rire coquin des légendes amérindiennes qui transcendent encore aujourd'hui les explications rationnelles du monde occidental par leur poésie et leur intériorité. Et qui revendiquent une place primordiale dans ces terres dont ils sont les premiers occupants, et dans cet espace symbolique qu'est la culture, dont ils sont peu à peu chassés. Les deux grands héros Baptiste et Carcajou, _ , car c'est bien de deux grands héros qu'il s'agit : historiquement, Baptiste étant sans doute en 1910 le premier héros vraiment canadien dans la $\mathrm{BD}$, et Carcajou étant un héros transposé du monde des légendes au monde de la $\mathrm{BD}$ - ces deux héros représentent l'ensemble de leurs lecteurs ou auditeurs. Ils expriment le rire complice.

Quant à Onésime et au Capitaine Kébec, ce sont deux antihéros. Le premier est le rural gaffeur et malchanceux. Le pauvre type sympathique est-il une métaphore du Québec traditionnel empêtré face à la montée de la modernité? Le second est avant tout un clin d'œil. Miroir ou reflet discordant des super antihéros loufoques canadiens et américains, son 
image très forte visuellement a imprégné l'imaginaire des lecteurs de BD tout d'abord, qui éprouvaient de la nostalgie pour ce petit bonhomme plus grand que nature, puis s'est hissée à la hauteur de l'un des symboles de la contre-culture en général. Il incarne la dérision d'un art de la surpuissance hérité de nos voisins du Sud qui se prétendent les rois du monde.

Ce qui unit les deux dernières œuvres, celles de Doucet et de Rabagliati, au-delà du fait que nous ne sommes plus dans le monde symbolique des héros, mais dans la peau d'individus qui sont en fait la représentation des auteurs eux-mêmes, est que désormais, l'Autre n'est plus clairement devant nous (le Canadien anglophone), mais à l'intérieur de nous. "Ainsi le Québécois veut être différent de l'Autre (souci de distinction) sans cesser de lui ressembler (souci d'équivalence ou de normalisation) » (Létourneau 18). Le multiculturalisme opère une fusion lente entre les valeurs des uns et des autres.

Dans la fable de Doucet, nous sommes aux antipodes de la complicité comique. Dans la ville contemporaine multiethnique non identifiée, qui pourrait être aussi bien Montréal que Toronto ou New York, la jeunesse se sent seule, abandonnée dans les méandres de la vie ici symbolisés par le métro. Les liens entre les personnes se mutent en corridors dans lesquels les gens de diverses nationalités évoluent pour se retrouver puis brusquement se quitter. Il y a une sensation de perte qui est fortement évoquée, perte de sens, perte de liens humains véritables, perte de repères dans un monde déshumanisé où plus rien n’indique le chemin : c'est la parabole du labyrinthe anonyme et angoissant. Et donc l'envers de l'identité, la non-identité? Quant à la ville de Rabliagliati, c'est la ville québécoise accueillante qui est chantée. Mais même si les caractéristiques de la ville de Québec sont bien présentes, les noms de rues et de villages environnants clairement situés, c'est davantage la géographie sociale qui est dépeinte. Et cette géographie n'est pas particulière au Québec, mais à son environnement plus large. C'est l'ensemble de son pays que Rabagliati dépeint en larges traits sensibles.

Associer ainsi des œuvres typiquement québécoises à une mentalité sous-jacente canadienne est un exercice de haute voltige qui pourrait prêter flanc à des critiques, de distorsion de la réalité culturelle par exemple. Pourtant, nous restons profondément convaincus au sortir de cette aventure, que les œuvres ici décrites partagent un héritage. Qu'elles soient intrinsèquement québécoises, quel que soit le sens que l'on donne à cette vaste notion, nous le clamons haut et fort. Mais, si on les compare à des œuvres de la BD européenne ou asiatique, on sent immédiatement cette couleur particulière qui les teinte. Cette couleur porte différents noms, nous avons fait le pari de l'appeler canadienne. 


\section{Bibliographie}

Bourgeois, Albéric. Les Voyages de Ladébauche autour du monde, Montréal : VLB Éditeur, 1982 (1910).

Canet, Raphaël. Nationalisme et société au Québec, Montréal : Athena, 2003.

Carpentier, André. "Occultation du réel et construction du "gag" dans Onésime ", Samson, J. et Al. (ed.), Colloque de BD de Montréal. Montréal : Analogon, 1985. 73-85.

Chartier, Albert. Les Aventures d'un Québécois typique, Onésime, tomes 1 et 2, Montréal : Éditions de l'Aurore, 1974.

Daveluy, Louise. "Les dessins et les mots pour dire... vrai? », Entre les lignes 2.1 (2005) : 32-33.

Deglise, Fabien. «Paul au parc. Personnage en quête de son moi.", Le Devoir 5 novembre 2011.

Doucet, Julie (2010), citée par Ladygunn. Ladygunn. 2010. Web. consulté août 2013.

Doucet, Julie. "Missing” in Dirty Plotte, Number five, Montréal : Drawn \& Quarterly, 1992.

Falardeau, Jean-Charles et Wade, Mason (ed.) La dualité canadienne, Toronto : University of Toronto Press, 1960.

Falardeau, Jean-Charles. Notre société et son roman, Montréal : HMH, 1967.

Falardeau, Mira. "La BD française est née au Canada en 1904.» Communications et langages 126 (2000) : 23-47.

Falardeau, Mira. Histoire de la bande dessinée au Québec, Montréal : VLB Éditeur, 2008.

Fournier Pierre. Les Aventures du Capitaine Kébec, Montréal : Éditions. Hydrocéphale Entêté, 1973.

Langlois, Simon (ed.). Identité et cultures nationales, Québec : Presses Université Laval, 1995.

Laniel, Christine et Lachapelle, Claude. Carcajou, le glouton fripon, Légendes indiennes attikamèques, s.l., Éditions Appartenance, 1982.

Létourneau, Jocelyn. Que veulent vraiment les Québécois?, Montréal : Boréal, 2006.

Mélançon, Claude. Légendes indiennes du Canada, Montréal : Édition du Jour, 1967.

Rabagliati, Michel. Paul à Québec, Montréal : La Pastèque, 2009.

Vastokas, Joan M. "Art autochtone», Sud des Grands Lacs et Vallée du Saint-Laurent, Encyclopédie Canadienne. Web. 20 août 2013.

Viau, Michel. BDQ. Répertoire des publications de bandes dessinées an Québec. Montréal : Mille Îles, 1999. 